\title{
M2K: I. A Jupiter-Mass Planet Orbiting the M3V Star HIP 79431'
}

\author{
Kevin Apps, ${ }^{2}$ Kelsey I. Clubb, ${ }^{3}$ Debra A. Fischer,,${ }^{3,4}$ Eric Gaidos,,${ }^{5}$ Andrew Howard, ${ }^{6}$ John A. Johnson, ${ }^{7}$ \\ Geoffrey W. Marcy, ${ }^{6}$ Howard Isaacson, ${ }^{6}$ Matthew J. Giguere, ${ }^{4}$ Jeff A. Valenti, ${ }^{8}$ \\ Victor Rodriguez, ${ }^{3}$ CARly Chubak, ${ }^{6}$ And Sebastien Lepine ${ }^{9}$ \\ Received 2009 December 10; accepted 2009 December 28; published 2010 January 12
}

\begin{abstract}
Doppler observations from Keck Observatory reveal the presence of a planet with $M \sin i$ of $2.1 M_{\text {Jup }}$ orbiting the M3V star HIP 79431. This is the sixth giant planet to be detected in Doppler surveys of $\mathrm{M}$ dwarfs and it is one of the most massive planets discovered around an $\mathrm{M}$ dwarf star. The planet has an orbital period of 111.7 days and an orbital eccentricity of 0.29 . The host star is metal rich, with an estimated $[\mathrm{Fe} / \mathrm{H}]=+0.4$. This is the first planet to emerge from our new survey of $1600 \mathrm{M}$-to-K dwarf stars.
\end{abstract}

\section{INTRODUCTION}

Doppler surveys for exoplanets are monitoring almost every bright, chromospherically inactive solar type star (late F, G, and early $\mathrm{K}$ types) and a fraction of the known $\mathrm{M}$ dwarfs within $30 \mathrm{pc}$. These successful programs have discovered about 400 exoplanets ranging in mass from a few times that of the Earth up to the lower mass limit for brown dwarfs (i.e., about $12 M_{\text {Jup }}$ ). This ensemble of exoplanets has revealed correlations between both the metallicity and mass of the host star with the occurrence rate of exoplanets (Santos et al. 2004; Fischer \& Valenti 2005; Lovis \& Mayor 2007; Johnson et al. 2007).

The occurrence rate of gas giant planets around $\mathrm{M}$ dwarfs appears to be lower than for F, G, and early K-type stars. Endl et al. (2003) detected only one planetary companion in a sample of $100 \mathrm{M}$ dwarf primaries. Johnson et al. (2007) carried out a statistical analysis and showed that late $\mathrm{K}$ and early $\mathrm{M}$ dwarf stars have a $1.8 \% \pm 1.0 \%$ occurrence rate for Jovian planets compared to $4.2 \% \pm 0.7 \%$ for solar-mass stars and $8.9 \% \pm$ $2.9 \%$ for higher-mass subgiants.

However, the protoplanetary disks of $\mathrm{M}$ dwarfs may still be a robust environment for the formation of lower-mass planets.

\footnotetext{
${ }^{1}$ Based on observations obtained at the W. M. Keck Observatory, which is operated by the University of California.

${ }^{2}$ 75B Cheyne Walk, Surrey RH6 7LR, UK.

${ }^{3}$ Department of Physics \& Astronomy, San Francisco State University, San Francisco, CA 94132.

${ }^{4}$ Department of Astronomy, Yale University, New Haven, CT 06511; debra. fischer@yale.edu.

${ }^{5}$ Department of Geology and Geophysics, University of Hawaii, Honolulu, HI 96822.

${ }^{6}$ Department of Astronomy, University of California, Berkeley, Berkeley, CA 94720.

${ }^{7}$ Department of Astronomy, California Institute of Technology, Pasadena, CA 91125.

${ }^{8}$ Space Telescope Science Institute, Baltimore, MD 21218.

${ }^{9}$ American Museum of Natural History, New York, NY 10023.
}

Forveille et al. (2009) find that $30 \%$ of the 20 known planets with $M \sin i<0.1 M_{\text {Jup }}$ orbit $\mathrm{M}$ dwarf stars. Consistent with this result, microlensing surveys (Sumi et al. 2009) find far more planets with masses in the range between a few to $20 M_{\oplus}$. These empirical results are compatible with core accretion models of planet formation. Ida \& Lin (2005) predict that gas accretion will be quenched by gap formation in disks with smaller aspect ratios; the low surface density in the protoplanetary disks of $\mathrm{M}$ dwarfs implies a low formation probability for gas giants because massive cores do not form before gas depletion. Laughlin et al. (2004) also surmise that a significant population of "failed Jupiters" with cores of a few Earth masses should exist around $\mathrm{M}$ dwarfs.

$\mathrm{M}$ dwarfs constitute the majority of stars populating our galaxy. Among the $\sim 150$ stars within $8 \mathrm{pc}$, about 120 are early-type M dwarfs, while only 15 are $\mathrm{G}$ dwarfs. Low-mass stars are attractive targets for searches of terrestrial mass planets within the habitable zone where liquid water might be present (Tarter et al. 2007; Gaidos et al. 2007). The habitable zone is closer to low luminosity stars and the shorter period planets induce larger, more easily detected reflex velocities. Yet, fewer than $10 \%$ of late $\mathrm{K}$ and early $\mathrm{M}$ dwarf stars within $30 \mathrm{pc}$ are being monitored by Doppler surveys (Mayor et al. 2009; Butler et al. 2006; Endl et al. 2003). These stars are challenging targets because they are intrinsically faint at optical wavelengths. Indeed, many late-type $\mathrm{M}$ dwarfs beyond $10 \mathrm{pc}$ are only now being detected; recently, the SUPERBLINK survey (Lepine 2005; Lepine \& Shara 2005) identified a few thousand $\mathrm{M}$ dwarfs closer than $30 \mathrm{pc}$, opening up the possibility for larger scale surveys of these stars.

\section{A NEW M AND K DWARF SURVEY}

To learn more about the rate of exoplanet occurrence for $\mathrm{M}$ dwarf stars and mass and stellar metallicity dependences, we have started a survey of 1600 late K and early M dwarfs. The target stars are drawn from the SUPERBLINK survey, which provides an all-sky census of stars with proper motion 


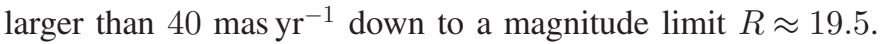
Main-sequence stars are identified based on their location in a reduced proper motion diagram. A fraction of the stars are listed in the Hipparcos catalog; for those stars we calculate the distance from their Hipparcos parallax. All other stars have their distances estimated based on the empirical $V, V-J$ colormagnitude relationship from Lepine (2005). We select as probable late $\mathrm{K}$ and $\mathrm{M}$ dwarfs all stars with optical-to-infrared color $V-J>2.75$, which roughly corresponds to absolute visual magnitudes $M_{v}>8.5$ and masses $M<0.6 M_{\odot}$. We further narrow down our sample to stars with photometric distances $d<50 \mathrm{pc}$ and visual magnitudes $V<12.5$. The low proper motion limit and short distance range minimizes the kinematic bias, and the sample is expected to be complete for stars with transverse velocities $v_{T}>9.5 \mathrm{~km} \mathrm{~s}^{-1}\left(v_{T}>5.7 \mathrm{~km} \mathrm{~s}^{-1}\right.$ for the $30 \mathrm{pc}$ subsample), which effectively samples the local disk population over the full range of typical stellar ages, as the selection even includes stars from nearby young moving groups (Lepine \& Simon 2009). Of the final sample of 1600 stars, $40 \%$ are listed in the Hipparcos catalog.

We carry out low-resolution spectroscopic screening of all candidates before they are observed at Keck using the Mark III spectrograph at the $1.3 \mathrm{~m} \mathrm{McGraw-Hill} \mathrm{Telescope} \mathrm{at} \mathrm{the}$ MDM Observatory at Kitt Peak. These observations are used to confirm the spectral type and main sequence status before beginning observations at Keck.

Modeled after the N2K program (Fischer et al. 2005), our program uses a quick-look strategy to obtain three or four nearly consecutive observations of target stars to flag short-period candidates. We then continue to obtain approximately log-spaced time series observations to detect longer period planets. Here, we present the first planet to emerge from this program.

\section{HIP 79431}

\subsection{Stellar Characteristics}

HIP 79431 is an M3V star with apparent magnitudes of $V=11.34, \quad J=7.56, \quad H=6.86, \quad K=6.59, \quad$ and color $B-V=1.486$. The Hipparcos parallax (ESA 1997; van Leeuwen 2007) of $69.46 \pm 3.12$ mas yields a distance of $14.4 \mathrm{pc}$ and we use this to calculate the absolute magnitudes $M_{V}=10.47$, $M_{J}=6.69, M_{H}=5.99, M_{K}=5.72$. The proper motions

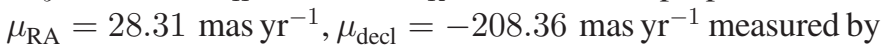
the Hipparcos mission (ESA 1997) and our measured absolute radial velocity of $-4.7 \mathrm{~km} \mathrm{~s}^{-1}$ correspond to the small space velocities of $U=+8.5 \mathrm{~km} \mathrm{~s}^{-1}, \quad V=+2.1 \mathrm{~km} \mathrm{~s}^{-1}, \quad W=$ $-4.8 \mathrm{~km} \mathrm{~s}^{-1}$.

Delfosse et al. (2000) have established mass-luminosity calibrations for low-mass stars and find low dispersion relations for $M_{J}, M_{H}$, and $M_{K}$. The average of these three infrared massluminosity relations yield an average stellar mass of $0.49 M_{\odot}$ for HIP 79431.
It is challenging to assess metallicity from $\mathrm{M}$ dwarf spectra, largely because of uncertainties in the molecular line data. Bonfils et al. (2005) first derived a photometric calibration for $\mathrm{M}$ dwarf metallicities and this work has been updated by Johnson \& Apps (2009). Both groups used spectral synthesis modeling to determine $[\mathrm{Fe} / \mathrm{H}]$ for bright $\mathrm{F}$ and G-type stars in binary systems with $\mathrm{M}$ dwarf companions and assigned the metallicity of the primary star to the M dwarf companion. Based on the star's height above the $M_{K}$ versus $V-K$ main sequence, the calibration of Johnson \& Apps (2009) yields a metallicity of $[\mathrm{Fe} / \mathrm{H}]=+0.4 \pm 0.1$.

The effective temperature for $\mathrm{M}$ dwarfs is usually derived from a color-temperature relation. The $K$-band temperature relation calibrated by Casagrande et al. (2008) is insensitive to metallicity and $\mathrm{M}$ dwarf fluxes peak near the infrared $K$ band. The Casagrande et al. (2008) relation has a scatter of only $19 \mathrm{~K}$ and yields $T_{\text {eff }}=3191 \mathrm{~K}$ for HIP 79431 . This is similar to the effective temperature of $3236 \mathrm{~K}$ that can be derived with the less robust $B-V$ calibration of Valenti \& Fischer (2005) for FGKtype stars.

Because the continuum flux near the $\mathrm{Ca}$ II $\mathrm{H} \& \mathrm{~K}$ lines is so low in $\mathrm{M}$ dwarfs, it is typical to see strong emission from the core of the Ca II H \& K lines. Isaacson \& Fischer (2010, in preparation) measure $S_{\mathrm{HK}}=1.15 \pm 0.06$ in this star. The $\log R_{\mathrm{HK}}^{\prime}$, activity-based rotation period, and ages are not calibrated for stars with $B-V$ greater than 1.0; however, Isaacson \& Fischer (2010, in preparation) find that this value of $S_{\mathrm{HK}}$ is in the fiftieth percentile for stars of this color, so the star does not have an unusually high level of chromospheric activity for a dwarf star with this $B-V$ color. The stellar characteristics are summarized in Table 1.

\subsection{Doppler Observations and Keplerian Fit}

HIP 79431 was added to the Keck program in 2009 April as part of our exoplanet survey of low-mass stars. We obtained 13 Doppler measurements of HIP 79431 over 6 months using the HIRES spectrometer (Vogt et al. 1994). Exposure times of $600 \mathrm{~s}$

TABLE 1

STELlar PARAMETERS: HIP 79431

\begin{tabular}{|c|c|}
\hline Parameter & \\
\hline$V \quad \ldots$ & 11.34 \\
\hline$M_{V} \quad \ldots \ldots \ldots \ldots \ldots \ldots \ldots \ldots \ldots \ldots \ldots$ & 10.47 \\
\hline$M_{K} \quad \ldots \ldots \ldots \ldots \ldots \ldots \ldots \ldots \ldots \ldots \ldots \ldots$ & 5.72 \\
\hline 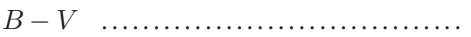 & 1.486 \\
\hline Spectral Type $\ldots \ldots \ldots \ldots$ & M3V \\
\hline Distance $(\mathrm{pc}) \ldots .$. & 14.4 \\
\hline$T_{\text {eff }} \quad \ldots \ldots \ldots \ldots \ldots$ & $3191(1000$ \\
\hline 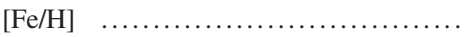 & $+0.4(0.1)$ \\
\hline 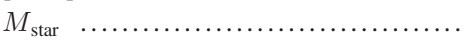 & $0.49(0.05)$ \\
\hline 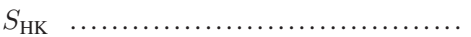 & $1.15(0.06)$ \\
\hline Radial Velocity $\left(\mathrm{km} \mathrm{s}^{-1}\right) \quad \ldots \ldots \ldots \ldots \ldots$ & -4.7 \\
\hline
\end{tabular}


for this $V=11.3$ star yielded a signal-to-noise ratio of just under 100 .

Our Doppler analysis makes use of an iodine absorption cell in the light path before the entrance slit of the HIRES spectrometer. The iodine absorption lines in each program observation are used to model the wavelength scale and the instrumental profile of the telescope and spectrometer optics for each observation (Marcy \& Butler 1992; Butler et al. 1996). The typical Doppler precision for this faint star is about $3 \mathrm{~km} \mathrm{~s}^{-1}$. Based on the rms scatter of other stars on our program, we have empirically derived an additional error which may arise from astrophysical noise sources or systematic uncertainties in our data. Figure 1 shows time series Doppler observations for stars with constant radial velocities and $B-V$ colors similar to HIP 79431. The typical rms scatter is about $2.5 \mathrm{~km} \mathrm{~s}^{-1}$. We adopt $2.5 \mathrm{~km} \mathrm{~s}^{-1}$ as representative of the combined radial velocity jitter and systematic errors for late-type stars and add this in quadrature with our formal single measurement errors when fitting and plotting our data.

The observation dates, radial velocity data, and measurement uncertainties for HIP 79431 are listed in Table 2. In this Table, the measurement uncertainties are not increased by the $2.5 \mathrm{~km} \mathrm{~s}^{-1}$ jitter term. The radial velocities exhibit an unambiguous signal. The radial velocities were fit with a Keplerian model and the uncertainties in the orbital parameters were derived using a bootstrap Monte Carlo analysis with 1000 realizations of the data

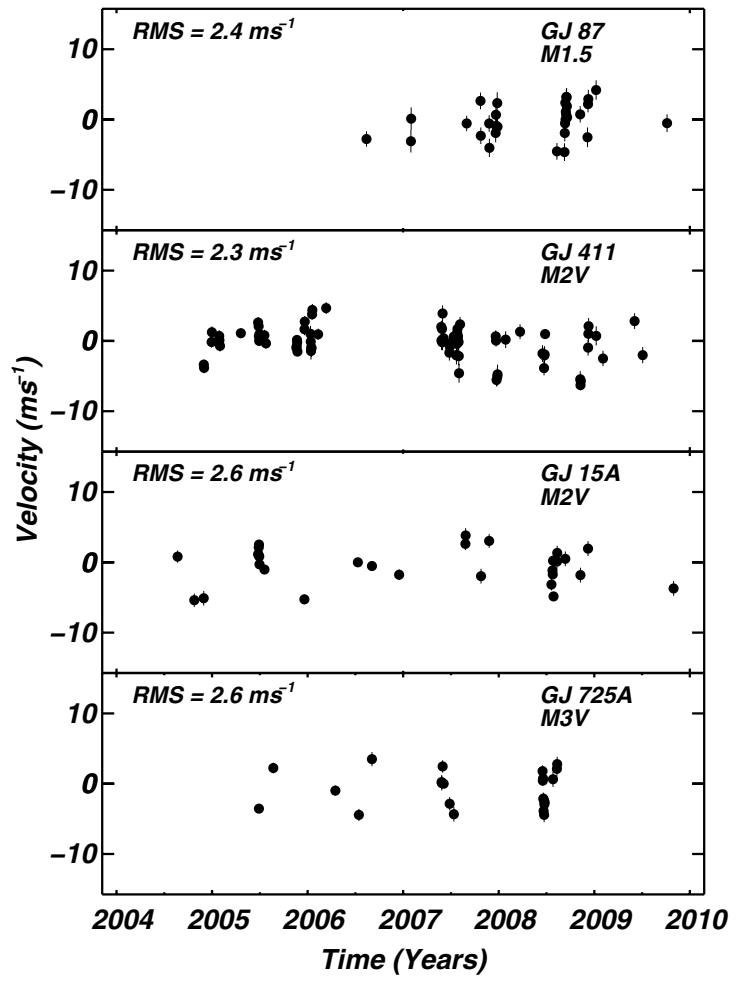

FIG. 1.-Doppler measurements of four M dwarf stars with constant radial velocities. These stars have an rms scatter of about $2.5 \mathrm{~m} \mathrm{~s}^{-1}$ and represent velocity precision typical of late-type stars on our program.
(Marcy et al. 2005) in the following way: first, the theoretical velocities from the best-fit Keplerian model are subtracted, then the residual velocities are randomized, added back to the theoretical velocities, and refit. This analysis captures the full range of uncertainties, including systematic errors; however, it will also scramble a signal from any additional planets, so it is only appropriate for good $\chi_{\nu}^{2}$ fit models.

The Keplerian fit yields an orbital period of $111.7 \pm 0.7$ days and a semivelocity amplitude of $149.5 \pm 2.5 \mathrm{~m} \mathrm{~s}^{-1}$. The stellar mass of $0.49 M_{\odot}$ implies a companion mass with $M \sin i=$ $2.1 M_{\text {Jup }}$ and a semimajor axis of $0.35 \mathrm{AU}$. The parameters for the Keplerian orbit are listed in Table 3.

The Doppler velocities are plotted in Figure 2 with a solid line indicating the best-fit Keplerian model. In this plot, the single measurement errors have been increased by adding a modest $2.5 \mathrm{~m} \mathrm{~s}^{-1}$ for stellar jitter and systematic errors. The rms of the residuals to our Keplerian model is $3.9 \mathrm{~m} \mathrm{~s}^{-1}$ and the $\chi_{\nu}^{2}$ fit is 0.84 , demonstrating that the fit is consistent with our single measurement errors and the added assumed jitter of $2.5 \mathrm{~m} \mathrm{~s}^{-1}$. As such a good fit implies, there is no evidence for additional planets.

\section{PLANET OR BROWN DWARF?}

Because the Doppler technique derives $M \sin i$ and not the total mass, this leaves open the question of whether this is actually a stellar binary system in a nearly face-on orbit. HIP 79431 is only 14.4 pc away and a stellar companion on a nearly face-on orbit would induce motion around the barycenter of order 10 mas. The reanalysis of the Hipparcos astrometry is consistent (goodness-of-fit parameter of 0.15 ) with the proper motion of a single star (van Leeuwen 2007). We used the intermediate astrometric data (ESA 1997) to place an upper limit on the companion mass as a function of confidence level (one minus the false alarm probability [FAP]). The abscissa residuals to the Hipparcos parallax and proper motion solution span

TABLE 2

RADIAL Velocities FOR HIP 79431

\begin{tabular}{|c|c|c|}
\hline JD 2,440,000 & $\begin{array}{c}\mathrm{RV} \\
\left(\mathrm{m} \mathrm{s}^{-1}\right)\end{array}$ & $\begin{array}{c}\sigma_{\mathrm{RV}} \\
\left(\mathrm{m} \mathrm{s}^{-1}\right)\end{array}$ \\
\hline 14928.07496 & -63.11 & 2.73 \\
\hline $14929.11611 \ldots$ & -70.45 & 2.84 \\
\hline $14984.87477 \quad \ldots \ldots$ & 103.92 & 2.68 \\
\hline $14986.96264 \quad \ldots$ & 125.53 & 3.14 \\
\hline 14987.94866 & 140.47 & 3.10 \\
\hline 15028.96005 & -7.06 & 3.88 \\
\hline 15041.89538 & -74.88 & 3.21 \\
\hline $15042.92741 \quad \ldots$ & -81.75 & 3.55 \\
\hline $15048.75289 \quad \ldots \ldots \ldots \ldots \ldots$ & -104.55 & 2.80 \\
\hline $15074.72733 \quad \ldots \ldots \ldots \ldots \ldots$ & -140.47 & 2.84 \\
\hline $15078.74506 \quad \ldots \ldots \ldots \ldots \ldots$ & -119.78 & 2.90 \\
\hline$\ldots \ldots \ldots \ldots \ldots$ & -64.40 & 2.97 \\
\hline $15106.73408 \quad \ldots \ldots \ldots \ldots \ldots$ & 139.86 & 3.52 \\
\hline
\end{tabular}


TABLE 3

Orbital PARAMETERS FOR HIP 79431B

\begin{tabular}{|c|c|}
\hline Parameter & \\
\hline Period (days) & $111.7(0.7)$ \\
\hline 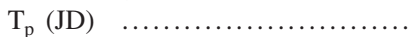 & $2,454,980.3(1.2)$ \\
\hline 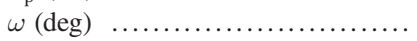 & $287.4(3.2)$ \\
\hline Eccentricity $\quad \ldots \ldots \ldots \ldots \ldots \ldots \ldots$ & $0.29(0.02)$ \\
\hline $\mathrm{K}_{1}\left(\mathrm{~m} \mathrm{~s}^{-1}\right) \quad \ldots \ldots \ldots \ldots \ldots \ldots \ldots \ldots$ & $149.5(2.5)$ \\
\hline$a_{\text {rel }}(\mathrm{AU}) \quad \ldots \ldots \ldots \ldots \ldots \ldots \ldots \ldots$ & 0.36 \\
\hline$M \sin i\left(M_{\mathrm{Jup}}\right) \quad \ldots \ldots \ldots \ldots \ldots \ldots \ldots \ldots$ & 2.1 \\
\hline Number of observations $\ldots \ldots \ldots \ldots$ & 13 \\
\hline $\mathrm{rms}\left(\mathrm{ms}^{-1}\right) \quad \ldots \ldots \ldots \ldots \ldots \ldots \ldots \ldots \ldots$ & 3.9 \\
\hline$\chi_{\nu}^{2} \quad \ldots \ldots \ldots \ldots \ldots \ldots \ldots \ldots \ldots \ldots \ldots$ & 0.84 \\
\hline
\end{tabular}

about 10 orbits and are plotted in Figure 3. There is no power in a Lomb-Scargle periodogram near the orbital period of 111 days in these data. Assuming Gaussian statistics, a companion more massive than $3 M_{\odot}$ can be ruled out with $90 \%$ confidence (Fig. 4), but the Hipparcos data by themselves cannot eliminate the possibility of a late-type $\mathrm{M}$ or brown dwarf companion. We can, however, rule out the possibility that the location of HIP 79431 above the main sequence is due to a nearly equally luminous companion, and therefore conclude that this location is consistent with the high stellar metallicity (Johnson \& Apps 2009).

Even a brown dwarf mass would require an improbable nearly face-on orbit. If we take the lower limit for stellar mass as $70 M_{\text {Jup }}$, then the maximum inclination $i$ for our system to surpass the stellar mass threshold is given by

$$
i_{70 \mathrm{Jup}}=\arcsin \frac{M \sin i}{70 M_{\mathrm{Jup}}} .
$$

The probability, $P$ (star), that this particular inclination was observed can be calculated under the assumption that the orientation of orbits is random as

$$
P(\text { star })=\cos (0)-\cos \left(i_{70 \text { Jup }}\right)=4 \times 10^{-4},
$$

where $i$ is the orbital inclination from equation (1). We can likewise calculate the probability that HIP $79431 \mathrm{~b}$ falls in the brown dwarf range. This time, the inclination limit is far less severe since the planet only needs to have a true mass of $12 M_{\mathrm{Jup}}$ :

$$
i_{12 \text { Jup }}=\arcsin \frac{M \sin i}{12 M_{\mathrm{Jup}}}
$$

$P($ brown dwarf $)=\cos \left(i_{70 \text { Jup }}\right)-\cos \left(i_{12 \text { Jup }}\right)=0.015$

So, in order for HIP $79431 \mathrm{~b}$ to be a stellar companion, an inclination of less than $2^{\circ}$ from face-on is required and that orientation has a probability of only $0.04 \%$. If HIP 79431 b is really a brown dwarf companion, the orbital inclination must be between $2^{\circ}-10^{\circ}$; the probability of this orientation is $1.5 \%$.

\section{TRANSIT EPHEMERIS}

Because HIP 79431b has a semimajor axis of $0.36 \mathrm{AU}$, the planet has a relatively low transit probability. The planet also resides in a moderately eccentric orbit that brings it as close as $0.25 \mathrm{AU}$ to the host star at periastron. For the orbit of HIP $79431 \mathrm{~b}, \omega=288 \pm 3$, so a primary transit would occur when the planet is close to apastron. This further reduces the probability of a primary transit and we calculate a transit probability of only $0.5 \%$. In this system, the secondary eclipse is somewhat more likely; without prior knowledge of a primary transit, we calculate a probability of $0.85 \%$ for the secondary eclipse.

If a transit were to occur, the transit depth would be remarkable. A planet with a mass of $2.1 M_{\text {Jup }}$ is supported by electron degeneracy and has a radius that is only a factor of 4 times smaller than the $\mathrm{M}$ dwarf host. We calculate a prospective primary transit depth of 98 millimags and transit duration of almost 7 hours. The probability for a secondary eclipse is about double that for the primary transit, however the photometric decrement would only be about 0.004 millimags.

Prospective transit times were predicted from the best-fit Keplerian parameters. The Thiele-Innes constants were used to project the orbit onto the plane of the sky and to determine the true anomaly corresponding to the central transit time, $T_{c}$. The time of the next $T_{c}$ is calculated to be 2010 February 21 at UT 18:17:23:92, with an uncertainty of $1.6 \mathrm{hr}$. The uncertainty in $T_{c}$ was determined by fitting the 1000 trials of Keplerian fits (used to derive uncertainties in the orbital parameters). For each trial, the central transit time, ingress, and egress were calculated and the standard deviation provided an estimate of the

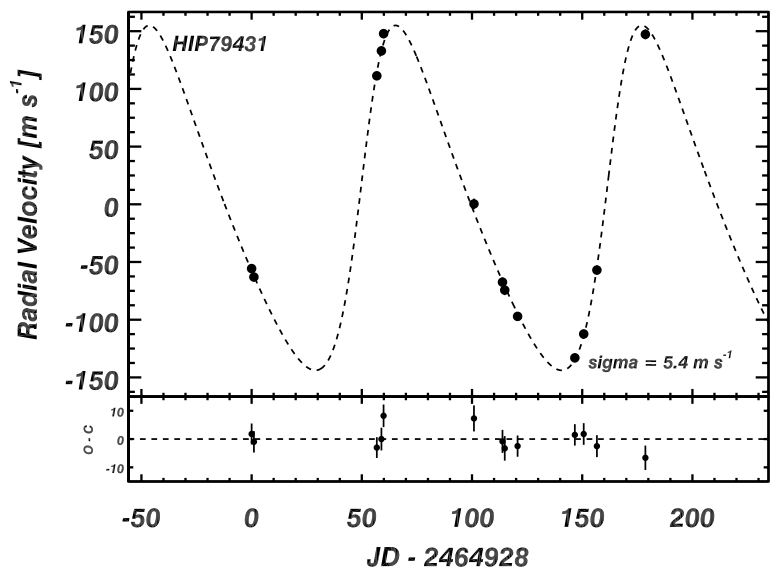

FIG. 2.- Time series radial velocities from Keck Observatory are plotted for HIP 79431 with $2.5 \mathrm{~m} \mathrm{~s}^{-1}$ of expected velocity jitter added in quadrature with the single measurement uncertainties. The Keplerian model is overplotted with an orbital period of 111 days, velocity amplitude of $149.5 \mathrm{~m} \mathrm{~s}^{-1}$, and eccentricity, $e=0.29$. With these parameters and the stellar mass of $0.49 M_{\odot}$, we derive a planet mass, $M \sin i=2.1 M_{\text {Jup }}$, and semimajor axis of $0.36 \mathrm{AU}$. 


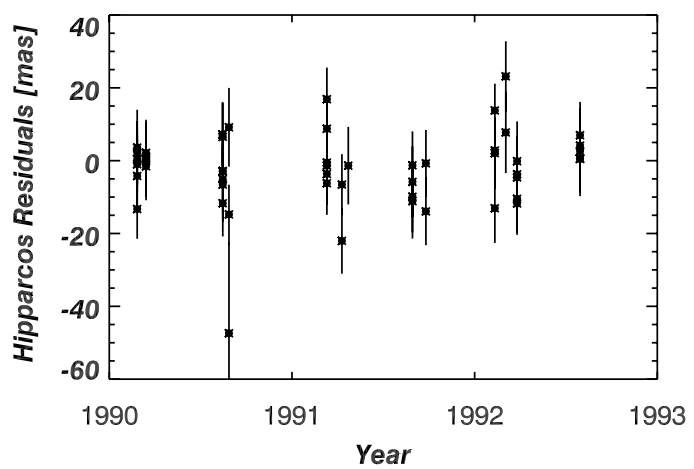

FIG. 3.-Astrometric residuals (unbinned) from the Hipparcos intermediate data after fitting for parallax and proper motion.

uncertainty. Future transit times can be estimated by rolling forward an integral number of orbital periods, however, uncertainty in $T_{c}$ increases with time from our last Doppler measurement.

\section{DISCUSSION}

As part of a new Doppler quick-look survey for late $\mathrm{K}$ and early $\mathrm{M}$ dwarfs, we have detected a planet with $M \sin i=2.1$ $M_{\text {Jup }}$ orbiting the M3V star, HIP 79431. The host star appears to be metal-rich with a photometrically determined $[\mathrm{Fe} / \mathrm{H}]=+0.4$.

Massive planets around late-type stars are easy detections. However, the sample of $\mathbf{M}$ dwarf stars with planets is relatively small; only six have detected gas giant planets ( $M \sin i=$ $0.5 M_{\text {Jup }}$ ). The remarkable system of planets orbiting GJ 876 (M4V) has three planets and two of these are gas giants in a 2:1 mean motion resonance; GJ $876 \mathrm{~b}$ has $M \sin i=$ $1.935 M_{\text {Jup }}$ and orbital period of about 30 days and GJ $876 \mathrm{c}$ has $M \sin i=0.619 M_{\text {Jup }}$ with an orbital period of 60 days (Marcy et al. 2001, 1998; Delfosse et al. 1998). GJ 849 (M3.5V) has a planet with $M \sin i=0.82 M_{\text {Jup }}$ in a $5 \mathrm{yr}$ orbit with significant residual velocities that now exhibit curvature, consistent with an additional planet in a wider orbit. GJ 317 (M3.5V) has a planet with $M \sin i=1.17 M_{\text {Jup }}$ with a period of about $1.9 \mathrm{yr}$ and residual velocities consistent with a Jupiter-mass planet in an orbit of more than $7 \mathrm{yr}$ (Johnson et al. 2007). GJ 832 (M1.5V) has a Jupiter-like planet in a 9.4 yr orbit (Bailey et al. 2009). GJ $179(\mathrm{M} 3.5 \mathrm{~V})$ has a Jupiter-mass planet in a $6.3 \mathrm{yr}$ orbit (Howard et al. 2009). GJ 649 (M1.5V) has a Saturn-mass planet in a $1.6 \mathrm{yr}$ orbit (Johnson et al. 2009).

It is curious that half of the known giant planets orbiting lowmass M dwarf stars reside in relatively wide orbits. Kennedy \& Kenyon (2009) find that grain growth time scales are limited by photo evaporation, with more rapid loss of disks in high mass stars. They note that the stellar mass-dependent disk dispersal timescales may account for the fact that planets orbiting highermass stars have wider orbits, since the disk may disperse more rapidly and halt inward migration. Perhaps lower surface den-

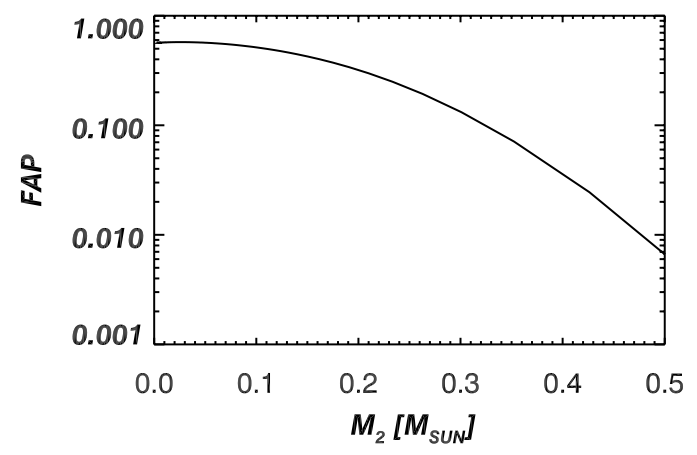

FIG. 4.-False-alarm probability (one minus the confidence level) of the companion exceeding a given mass, given the Hipparcos abscissa residuals for HIP 79431.

sity creates an analogous migration-hindering environment for low-mass stars. Or, perhaps the protoplanetary disk of $\mathrm{M}$ stars forms fewer planets so that planet-planet scattering events are less common. With less competition for raw materials in the disk, it might be possible for single or well-spaced giant planets to dominate at wide separations. This is also a domain where gravitational instability could play a role (Boss 2006).

HIP $79431 \mathrm{~b}$ is one of the most massive planets detected in orbit around an M dwarf star. The calculated probability that this is actually an equal-mass stellar companion is vanishingly small: $4 \times 10^{-4}$. The Hipparcos astrometric data do not rule out the possibility that this is a low-mass star or brown dwarf companion, but we calculate only a $1.5 \%$ probability that HIP $79431 \mathrm{~b}$ has an orbital inclination less than $10^{\circ}$ and a true mass greater than $12 M_{\text {Jup }}$. Statistically, this leaves a $98.5 \%$ probability that HIP $79431 \mathrm{~b}$ is a planet with a mass between 2.1 and $12 M_{\text {Jup }}$.

The Keplerian motion induced by a single planet can explain our data, although much less massive planets may exist on interior or exterior orbits. The excellent agreement of the singleplanet Keplerian planet solution with the data limits the mass of any second planet in the system. We performed a linear perturbation analysis around the one-planet solution and found that we can rule out planets more massive than $M_{3} \sin i$ of 12 or 15 Earths near the $3: 1$ or $2: 1$ interior resonances, respectively.

Although Doppler surveys show that massive companions to M dwarf stars are rare, evidence for a few Jupiter-mass planets candidates has emerged from microlensing surveys that sample wider orbits. (Because low mass stars dominate the galactic population, they are common lenses for background stars.) Gaudi et al. (2002) place an upper limit and find that fewer than $33 \%$ of $\mathrm{M}$ dwarfs have Jupiter-mass companions between 1.5 and 4 AU. Nevertheless, only a small number of Jupiter-mass planets have been detected in microlensing surveys (Bond et al. 2004; Udalski 2005; Gaudi et al. 2008; Dong et al. 2009).

We gratefully acknowledge the dedication and support of the Keck Observatory staff, especially Grant Hill and Scott Dahm 
for support of HIRES and Greg Wirth for support of remote observing. D. A. F. acknowledges research support from NASA grant NNX08AF42G as well as NASA support through the Keck PI Data Analysis Fund. E. G. acknowledges support by NSF grant AST0908419. A. W. H. gratefully acknowledges support from a Townes Postdoctoral Fellowship at the University of California, Berkeley Space Sciences Laboratory.

Data presented herein were obtained at the W. M. Keck Observatory from telescope time allocated to the National Aeronautics and Space Administration through the agency's scientific partnership with the California Institute of Technology and the
University of California. The Observatory was made possible by the generous financial support of the W. M. Keck Foundation. The authors wish to recognize and acknowledge the very significant cultural role and reverence that the summit of Mauna Kea has always had within the indigenous Hawaiian community. We are most fortunate to have the opportunity to conduct observations from this mountain. We also thank the University of Hawaii TAC for allocation of telescope time. This research has made use of the SIMBAD database, operated at CDS, Strasbourg, France, and of NASA's Astrophysics Data System Bibliographic Services.

\section{REFERENCES}

Bailey, J., Butler, R. P., Tinney, C. G., Jones, H. R. A., O’Toole, S., Carter, B. D., \& Marcy, G. W. 2009, ApJ, 690, 743

Bond, I., et al. 2004, ApJ, 606, 155 2005, A\&A, 442, 635

Boss, A. 2006, ApJ, 643, 501

Butler, R. P., Johnson, J. A., Marcy, G. W., Wright, J. T., Vogt, S. S., \& Fischer, D. A. 2006, PASP, 118, 1685

Butler, R. P., Marcy, G. W., Williams, E., McCarthy, C., Dosanjh, P., \& Vogt, S. S. 1996, PASP, 108, 500

Casagrande, L., Flynn, C., \& Bessell, M. 2008, MNRAS, 389, 585

Delfosse, X., Forveille, T., Segransan, D., Beuzit, J.-L., Udry, S., Perrier, C., \& Mayor, M. 2000, A\&A, 364, 217

Delfosse, X., Forveille, T., Mayor, M., Perrier, C., Naef, D., \& Queloz, D. 1998, A\&A, 338, 67

Dong, S., et al. 2009, ApJ, 698, 1826

Endl, M., Cochran, W. D., Tull, R. G., \& MacQueen, P. J. 2003, AJ, 126, 3099

ESA 1997, The Hipparcos and Tycho Catalogs (ESA-SP 1200; Noordwijk: ESA)

Fischer, D. A., \& Valenti, J. A. 2004, ApJ, 622, 1102

Fischer, D. A., et al. 2005, ApJ, 620, 481

Forveille, T., et al. 2009, A\&A, 493, 645

Gaidos, E., Haghighipour, N., Algol, E., Latham, D., Raymond, S., \& Rayner, J. 2007, Science, 318, 210

Gaudi, B. S., et al. 2008, Science, 319, 927 2002, ApJ, 566, 463

Howard, A., et al. 2009, ApJ, in press

Ida, S., \& Lin, D. N. C. 2005, ApJ, 626, 1045
Johnson, J., Howard, A. W., Marcy, G. W., Bowler, B. P., Henry, G. W., Fischer, D. A., Apps, K., Isaaccson, H., \& Wright, J. T. 2009, PASP, in press (2009arXiv0912.2730J)

Johnson, J. A., \& Apps, K. A. 2009, ApJ, 699, 933

Johnson, J. A., Fischer, D. A., Marcy, G. W., Wright, J. T., Driscoll, P., Butler, R. P., Hekker, S., Reffert, S., \& Vogt, S. S. 2007, ApJ, 665,785

Kennedy, G. M., \& Kenyon, S. J. 2009, ApJ, 695, 1210

Laughlin, G., Bodenheimer, P., \& Adams, F. C. 2004, ApJ, 612, L73

Lepine, S., \& Simon, M. 2009, AJ, 137, 3632

Lepine, S. 2005, AJ, 130, 1680

Lepine, S., \& Shara, M. M. 2005, AJ, 129, 1483

Lovis, C., \& Mayor, M. 2007, A\&A, 472, 657

Marcy, G. W., Butler, R. P., Vogt, S. S., Fischer, D. A., Henry, G. W., Laughlin, G., Wright, J. T., \& Johnson, J. A. 2005, ApJ, 619, 570

Marcy, G. W., Butler, R. P., Fischer, D. A., Vogt, S. S., Lissauer, J., \& Rivera, E. 2001, ApJ, 556, 296

Marcy, G. W., Butler, R. P., Vogt, S. S., Fischer, D. A., \& Lissauer, J. 1998, ApJ, 505, 147

Marcy, G. W., \& Butler, R. P. 1992, PASP, 104, 270

Mayor, M., et al. 2009, A\&A, 507, 487

Sumi, T., et al. 2009, preprint ( $\operatorname{arXiv0912.1171)}$

Santos, N. C., Israelian, G., \& Mayor, M. 2004, A\&A, 415, 1153

Tarter, J., et al. 2007, Astrobiology, 7, 30

Udalski, A., et al. 2005, ApJ, 628, 109

van Leeuwen, F. 2007, A\&A, 474, 653

Valenti, J., \& Fischer, D. A. 2005, ApJS, 159, 141

Vogt, S. S., et al. 1994, Proc. SPIE, 2198, 362 